\title{
APPROXIMATION OF THE BERGMAN NORM BY THE NORMS OF THE DIRECT PRODUCT OF TWO SZEGÕ SPACES
}

\author{
SABUROU SAITOH
}

ABstract. Let $G$ be a bounded regular region in the plane. Let $H_{2}^{1 / 2}(G)$ denote the Szegö space of $G$ composed of analytic functions on $G$ with finite norm

$$
\left\{\frac{1}{2 \pi} \int_{\partial G}|f(z)|^{2}|d z|\right\}^{1 / 2}<\infty .
$$

We set $f(z)=\sum_{j-1}^{\infty} \varphi_{j}(z) \psi_{j}(z)\left(\varphi_{j}, \psi_{j} \in H_{2}^{1 / 2}(G)\right)$. Then, we determine a necessary and sufficient condition for $f(z)$ to make the equality

$$
\begin{aligned}
& \frac{1}{\pi} \iint_{G}|f(z)|^{2} d x d y \\
& \quad=\min \left\{\sum_{j=1}^{\infty} \sum_{k=1}^{\infty} \frac{1}{2 \pi} \int_{\partial G} \varphi_{j}\left(z_{1}\right) \overline{\varphi_{k}\left(z_{1}\right)}\left|d z_{1}\right| \frac{1}{2 \pi} \int_{\partial G} \psi_{j}\left(z_{2}\right) \overline{\psi_{k}\left(z_{2}\right)}\left|d z_{2}\right|\right\}
\end{aligned}
$$

hold. The minimum is taken here over all analytic functions $\sum_{j=1}^{\infty} \varphi_{j}\left(z_{1}\right) \psi_{j}\left(z_{2}\right)$ on $G \times G$ satisfying $f(z)=\sum_{j=1}^{\infty} \varphi_{j}(z) \psi_{j}(z)$ on $G$.

1. Introduction and statement of result. Let $G$ denote an $N$-ply connected bounded regular region in the plane with boundary contours $\left\{C_{\nu}\right\}_{\nu=1}^{N}$. Let $B(G)$ and $H_{2}^{1 / 2}(G)$ denote the Bergman space and the Szegô space of $G$ composed of analytic functions on $G$ with finite norms

$$
\left\{\iint_{G}|f(z)|^{2} d x d y\right\}^{1 / 2}<\infty \quad(z=x+i y)
$$

and

$$
\left\{\frac{1}{2 \pi} \int_{\partial G}|f(z)|^{2}|d z|\right\}^{1 / 2}<\infty,
$$

respectively. In the latter case, $f(z)$ means the Fatou boundary value of $f$ at $z \in \partial G$. Let $\left\{Z_{p}(z) d z\right\}_{v=1}^{N-1}$ be a basis of analytic differentials on $\bar{G}$ which are real along $\partial G$ such that

$$
Z_{v}(z)=\int_{C_{\nu}} L(\zeta, z) d \zeta
$$

Received by the editors June 15, 1978.

AMS (MOS) subject classifications (1970). Primary 30A31; Secondary 30A04, 30A40.

Key words and phrases. Bergman and Szegö kernels, the direct product of two Szegö spaces, Dirichlet norm, approximation of the Bergman norm, generalized isoperimetric inequalities. 
$L(\zeta, z)$ is the adjoint $L$-kernel of the usual Bergman kernel $K(\zeta, z)$ on $G$. Cf. [6, Chapter 4]. Let $K^{E}(z, \bar{u})$ and $\hat{K}(z, \bar{u})$ denote the exact Bergman kernel and the Szegó kernel of $G$, respectively. Then, we have the identity

$$
4 \pi \hat{K}(z, \bar{u})^{2}=K^{E}(z, \bar{u})+\sum_{\nu=1}^{N-1} \sum_{\mu=1}^{N-1} C_{\nu, \mu} \overline{Z_{\nu}(u)} Z_{\mu}(z) \text { on } G
$$

for some uniquely determined constants $\left\{C_{v, \mu}\right\}$. D. A. Hejhal [2] established the positive definiteness of the real symmetric matrix $\left\|C_{\nu, \mu}\right\|$ by means of the Riemann theta function. For an elementary proof and a general result for that positive definiteness, see [3].

In [4], we obtained the following theorem:

TheOREM A [4, TheOREM 2.1]. Any $f(z) \in B(G)$ can be represented by a series

$$
f(z)=\sum_{j=1}^{\infty} \varphi_{j}(z) \psi_{j}(z) \quad\left(\varphi_{j}, \psi_{j} \in H_{2}^{1 / 2}(G)\right)
$$

and the inequality

$$
\begin{aligned}
& \frac{1}{\pi} \iint_{G}|f(z)|^{2} d x d y \\
& \quad<\min \left\{\sum_{j=1}^{\infty} \sum_{k=1}^{\infty} \frac{1}{2 \pi} \int_{\partial G} \varphi_{j}\left(z_{1}\right) \overline{\varphi_{k}\left(z_{1}\right)}\left|d z_{1}\right| \frac{1}{2 \pi} \int_{\partial G} \psi_{j}\left(z_{2}\right) \overline{\psi_{k}\left(z_{2}\right)}\left|d z_{2}\right|\right\}
\end{aligned}
$$

is valid. The minimum is taken here over all analytic functions $\sum_{j=1}^{\infty} \varphi_{j}\left(z_{1}\right) \psi_{j}\left(z_{2}\right)$ on $G \times G$ satisfying (1.1).

Conversely, if the jk sum in (1.2) is finite, then the function $f(z)$ defined by the series (1.1) belongs to the class $B(G)$.

In order to state a sense of the $j k$ sum in (1.2), we introduce the direct product

$$
H=H_{2}^{1 / 2}(G) \otimes H_{2}^{1 / 2}(G)
$$

of the two Szegõ spaces. Let $\left\{\Phi_{j}(z)\right\}_{j=1}^{\infty}$ be a complete orthonormal system of $H_{2}^{1 / 2}(G)$. Then, the $H$ is formed by functions on $G \times G$ such that

$$
f\left(z_{1}, z_{2}\right)=\sum_{j=1}^{\infty} \sum_{k=1}^{\infty} A_{j, k} \Phi_{j}\left(z_{1}\right) \Phi_{k}\left(z_{2}\right), \quad \sum_{j=1}^{\infty} \sum_{k=1}^{\infty}\left|A_{j, k}\right|^{2}<\infty
$$

and the scalar product $(,)_{H}$ of $H$ is defined as follows:

$$
\left(f\left(z_{1}, z_{2}\right), h\left(z_{1}, z_{2}\right)\right)_{H}=\sum_{j=1}^{\infty} \sum_{k=1}^{\infty} A_{j, k} \overline{B_{j, k}},
$$

where $h\left(z_{1}, z_{2}\right)=\sum_{j=1}^{\infty} \sum_{k=1}^{\infty} B_{j, k} \Phi_{j}\left(z_{1}\right) \Phi_{k}\left(z_{2}\right) \in H$. The $j k$ sum in (1.2) is the square of the norm of $f\left(z_{1}, z_{2}\right)$ in $H$. Cf. [1].

Next, we introduce the Hilbert space $H_{R}$ which is formed by restricting the functions in $H$ to the diagonal set $D$ of $G \times G$ formed by all the elements 
$\{(z, z) ; z \in G\}$. Here, for any such restriction $f \in H_{R}$, the norm $\|f\|_{R}$ is defined by $\min \|h\|_{H}$ for all $h \in H$, the restriction of which to $D$ is $f$. Of course, \|\|$_{H}$ denotes the norm of $H$. Cf. [1, Theorem II, p. 361]. Hence, the minimum in (1.2) is the square of the norm of $f$ in $H_{R}$. As a result of the general theory, we obtained the following:

TheOREM B [4, TheOREM 3.1, THE FirST PART]. In (1.2), equality holds for $f(z) \in B(G)$ if and only if

$$
(f(z), g(z))_{R}=0 \text { for all } g(z) \in B_{\text {real }}(G) .
$$

Here $(,)_{R}$ denotes the scalar product of $H_{R}$ and $B_{\text {real }}(G)$ the vector space generated by $\left\{Z_{\nu}(z)\right\}_{\nu=1}^{N-1}$.

In this paper, we show that

THEOREM 1.1. In (1.2), equality holds for $f(z) \in B(G)$ if and only if $f(z) d z$ is exact.

Therefore this paper also gives supplementary remarks to [4]. In the proof of this theorem, the following theorem is important:

ThEOREM C [5, TheOREM 2.1]. For any

$$
f\left(z_{1}, z_{2}\right)=\sum_{j=1}^{\infty} \sum_{k=1}^{\infty} A_{j, k} \Phi_{j}\left(z_{1}\right) \Phi_{k}\left(z_{2}\right) \in H,
$$

the restriction $f(z, z)$ to $D$ can be uniquely decomposed as follows:

$$
f(z, z)=h^{\prime}(z)+\sum_{\nu=1}^{N-1} d_{\nu} Z_{\nu}(z) \text { on } G,
$$

$h^{\prime}(z) \in B(G)$ and $\left\{d_{\nu}\right\}$ are constants and the inequality

$$
\begin{gathered}
\sum_{j=1}^{\infty} \sum_{k=1}^{\infty}\left|A_{j, k}\right|^{2} \\
>\min \left\{\sum_{j=1}^{\infty} \sum_{k=1}^{\infty} \frac{1}{2 \pi} \int_{\partial G} \varphi_{j}\left(z_{1}\right) \overline{\varphi_{k}\left(z_{1}\right)}\left|d z_{1}\right| \frac{1}{2 \pi} \int_{\partial G} \psi_{j}\left(z_{2}\right) \overline{\psi_{k}\left(z_{2}\right)}\left|d z_{2}\right|\right\} \\
=\frac{1}{\pi} \iint_{G}\left|h^{\prime}(z)\right|^{2} d x d y+\sum_{\nu=1}^{N-1} \sum_{\mu=1}^{N-1} D_{\nu, \mu} d_{\nu} \bar{d}_{\mu}, \\
\left\|D_{\nu, \mu}\right\| \text { is the inverse of }\left\|C_{\nu, \mu}\right\|
\end{gathered}
$$

is valid. The minimum is taken here over all analytic functions $\sum_{j=1}^{\infty} \varphi_{j}\left(z_{1}\right) \psi_{j}\left(z_{2}\right)$ on $G \times G$ satisfying

$$
f(z, z)=\sum_{j=1}^{\infty} \varphi_{j}(z) \psi_{j}(z) \text { on } G, \quad \varphi_{j}, \psi_{j} \in H_{2}^{1 / 2}(G) .
$$

2. Proof of Theorem. For any $f(z)=\sum_{j=1}^{\infty} \varphi_{j}(z) \psi_{j}(z)=h^{\prime}(z)+$ $\sum_{\nu=1}^{N-1} d_{v} Z_{v}(z) \in B(G)\left(\varphi_{j}, \psi_{j} \in H_{2}^{1 / 2}(b G)\right)$, from Theorem A and Theorem B, 
we have

$$
\begin{aligned}
& \frac{1}{\pi} \iint_{G}|f(z)|^{2} d x d y \\
& \quad=\frac{1}{\pi} \iint_{G}\left|h^{\prime}(z)+\sum_{\nu=1}^{N-1} d_{\nu} Z_{\nu}(z)\right|^{2} d x d y \\
& \quad<\min \left\{\sum_{j=1}^{\infty} \sum_{k=1}^{\infty} \frac{1}{2 \pi} \int_{\partial G} \varphi_{j}\left(z_{1}\right) \overline{\varphi_{k}\left(z_{1}\right)}\left|d z_{1}\right| \frac{1}{2 \pi} \int_{\partial G} \psi_{j}\left(z_{2}\right) \overline{\psi_{k}\left(z_{2}\right)}\left|d z_{2}\right|\right\} \\
& \quad=\frac{1}{\pi} \iint_{G}\left|h^{\prime}(z)\right|^{2} d x d y+\sum_{\nu=1}^{N-1} \sum_{\mu=1}^{N-1} D_{\nu, \mu} d_{\nu} \overline{d_{\mu}} .
\end{aligned}
$$

Since

$$
\iint_{G} h^{\prime}(z) \overline{Z_{\nu}(z)} d x d y=0 \text { for } \nu=1,2, \ldots, N-1,
$$

(cf. [6, Chapter 4]), we thus have

$$
\sum_{\nu=1}^{N-1} \sum_{\mu=1}^{N-1}\left(D_{\nu, \mu}-\iint_{G} Z_{\nu}(z) \overline{Z_{\mu}(z)} d x d y\right) d_{\nu} \overline{d_{\mu}}>0 .
$$

Suppose that for $f(z) \in B(G)$, equality holds in (1.2) and so in (2.1), then from Theorem $B$ and Theorem $C$, we have

$$
\begin{aligned}
(f(z), g(z))_{R} & =\left(h^{\prime}(z)+\sum_{\nu=1}^{N-1} d_{\nu} Z_{\nu}(z), g(z)\right)_{R} \\
& =\left(\sum_{\nu=1}^{N-1} d_{\nu} Z_{\nu}(z), g(z)\right)_{R} \\
& =0 \text { for all } g(z) \in B_{\text {real }}(G) .
\end{aligned}
$$

Hence all the $d_{\nu}$ are zero and so we have the desired result.

In particular, in the above proof, we obtained the following theorem. Cf. [2, Theorem 39, p. 107]:

THEOREM 2.1. The matrix

$$
\left\|D_{\nu, \mu}-\iint_{G} Z_{\nu}(z) \overline{Z_{\mu}(z)} d x d y\right\|^{(N-1)(N-1)}
$$

is positive definite.

\section{REFERENCES}

1. N. Aronszajn, Theory of reproducing kernels, Trans. Amer. Math. Soc. 68 (1950), 337-404.

2. D. A. Hejhal, Theta functions, kernel functions and Abeian integrals, Mem. Amer. Math. Soc. 129 (1972). 
3. S. Saitoh, The exact Bergman kernel and the kernels of Szegö type, Pacific J. Math. 71 (1977), 545-557.

4. __ The Bergman norm and the Szegö norm, Trans. Amer. Math. Soc. (to appear).

5. The Dirichlet norm and the norm of Szegö type (submitted).

6. M. Schiffer and D. C. Spencer, Functionals of finite Riemann surfaces, Princeton Univ. Press, 1954.

Dipartident of Mathiachtics, Faculty of Engnebrino, Guna Univirstry, 1-5-1, TENJIN-Cho, KIRYU 376, JAPAN 